Abbas, T. c102 Abdel-Razek, W. c102

Acikel, C. c115

Akiyama, S. c107

Amini, M. c73

Aminian, T. c73

Aminorroaya, A. c73

Aucella, F. c31

Bakoyannis, C. c37

Balakrishnan, V.S. c56

Baron, G. c63

Bastounis, E. c37

Bingol, N. c115

Bingol, S. c115

Bisceglia, L. c31

Brandi, L. c128

Burden, R. c86

Cabantchik, I. c63

Caglar, K. c115

Chang, C.-L. c122

Chen, C.-J. c122

Chen, C.-P. c122

Chen, L.-R. c122

Cui, Z c49

Cunningham, J. c1

Dasgupta, I. c86

De Bonis, P. c31

D'Errico, M. c31

Driss, F. c63
Duan, H. c92

Egfjord, M. c128

El-Agroudy, A. c97

El-Askalani, H. c102

El-Husseini, A. c97

Elias, M. c13

Eyileten, T. c115

Fan, S.L.S. c1

Filis, K. c37

Gatta, G. c31

Gejyo, F. c8

Georgopoulos, S. c37

Gharavi, M. c73

Giorgio, G. di c31

Goldstein, L.H. c13

Gotoh, H. c107

Gotoh, T. c107

Gotoh, Y. c107

Grandchamp, B. c63

Hassan, N. c97

Herrmann, W. c42

Higaki, Y. c18

Higashihara, E. c18

Ho, C.-S. c68

Horie, S. c18

Hosoya, T. c18

Huang, M.-M. c122

Inagaki, M. c107

Jaber, B.L. c56
Janghorbani, M. c73

Jaroszyński, A.J. c24

Kallenbach, M. c92

Kameda, S. c8

Kamura, K. c18

Katsahian, S. c63

Kazama, J.J. c8

Khalil, A. c97

Kilic, S. c115

Kirsch, C.-M. c42

Klonaris, C. c37

Kolta, A. c63

Książek, A. c24

Kuhlmann, M. c42

Laio, C.-S. c122

Lam, C.W.-K. c68

Lameire, N. c61

Law, W.-K. c68

Lee, C.-C. c122

Leung, C.-B. c68

Li, P.K.-T. c68

Liangos, O. c56

Lin, S.-M. c122

Maruyama, H. c8

Mentré, F. c63

Michel, C. c63

Mignon, F. c63

Mizutani, T. c78

Mochizuki, T. c18
Muscarella, L.A. c31

Nakashima, A. c78

Nakayama, T. c18

Narita, I. c8

Nutahara, K. c18

Obeid, R. c42

Oguchi, K. c107

Olgaard, K. c128

Papalambros, E. c37

Papapetrou, A. c37

Pereira, B.J.G. c56

Rao, M. c56

Rezvanian, H. c73

Ring, T. c92

Rosenfeld, T. c13

Sabry, A. c97

Salem, A. c97

Saliba, W. c13

Sedrati, N. c63

Selim, A. c102

Sheashaa, H. c97

Shigematsu, T. c8

Shih, Y.-L. c122

Shimizu, T. c18

Shneor, A. c13

Sobh, M. c102

Sonmez, A. c115

Stallone, C. c31

Steddon, S.J. c1
Swae'ed, S. c13

Szeto, C.-C. c68

Takasugi, N. c78

Tschen, S.-Y. c122

Tsuchiya, K. c18

Tsuji, M. c107

Turgman, Y. c13

Ueno, T. c78

Van Biesen, W. c61

Vanholder, R. c61

Vigilante, M. c31

Vourliotakis, G. c37

Vrtovsnik, F. c63

Vural, A. c115

Wang, C.-H. c122

Wang, H. c49

Xin, G. c49

Yamagata, Z. c78

Yamamoto, N. c18

Yamamoto, S. c8

Yenicesu, M. c115

Yilmaz, M.I. c115

Yorioka, N. c78

Załuska, W.T. c24

Zelante, L. c31

Zhao, M. c49

\title{
Subject Index Vol. 99, 2005
}

Actinin-4 c31

Acute renal failure c56

Adiponectin c115

Adynamic bone disease $\mathrm{c} 1$

Anemia c97

Angiography c13

Angiotensin II receptor blocker c18

Anti-CD20 (rituximab) c92

Anti-glomerular basement membrane disease c49

Antineutrophil cytoplasmic antibody c92

Antioxidants c13

Arrhythmias c24

Autosomal dominant polycystic kidney disease $\mathrm{c} 18$

Blood pressure control c86

- transfusion c122

Bone metabolism markers c78

- mineral density c78

- remodelling $\mathrm{cl}$
Calcium c24

Captopril c73

China, anti-glomerular basement membrane disease $\mathrm{c} 49$

Chronic renal failure c86

Continuous ambulatory peritoneal dialysis c86

Contrast nephropathy $\mathrm{c} 13$

Critical limb ischemia c37

Cross-linked N-terminal telopeptide c78

$\mathrm{Cu} / \mathrm{Zn}$ superoxide dismutase mRNA c107

Cyclosporin A c68

Denaturing high-performance liquid chromatography c31

Diabetes mellitus c73, c115

End-stage renal disease c37

Erythropoietin c102

Extracellular body water c24

Focal segmental glomerulosclerosis c31
Goodpasture syndrome c49

Hemodialysis c8, c56, c86

-, $\mathrm{Cu} / \mathrm{Zn}$ superoxide dismutase induction $\mathrm{c} 107$

Hepatitis $\mathrm{E}$ virus infection $\mathrm{c} 122$

Holotranscobalamin c42

Homocysteine c42

Hypertension c86

Immunoradiometric PTH assay c128

Infrainguinal bypass c37

Insulin sensitivity c115

Intermittent hemodialysis c56

Iron gluconate c97

-, non-transferrin-bound c63

$\mathbf{K} /$ DOQI guidelines c8

Methylmalonic acid c42

Mononuclear cells c42

Nandrolone decanoate c102

Osteodystrophy c1
Outcome predictors, acute renal failure c56

Oxidative stress c107

Parathyroid hormone c8, c128

- - , intact c8

- $\quad$, whole c128

Parenteral iron saccharate c97

Pentoxifylline c73

Phosphorus c1

Podocin c31

Potassium c24

Proteinuria c73

QT dispersion c24

Renal osteodystrophy c78

Rituximab c92

Secondary hyperparathyroidism c8

Type 1 collagen c78

Urine volume c56

Vitamin D c1, c128

Wegener's granulomatosis c92

\section{KARGER}

(C) 2005 S. Karger AG, Basel

Fax + 41613061234

E-Mail karger@karger.ch

www.karger.com 